\title{
Força muscular, funcionalidade e distância percorrida em pacientes com doença pulmonar obstrutiva crônica
}

\author{
Muscle strength, functionality and distance \\ traveled in patients with chronic obstructive \\ pulmonary disease
}

\author{
Luciano Xavier Gomes ${ }^{1}$ (D) \\ David Mendonça Santos ${ }^{2}$ (b) \\ Débora do Nascimento Santos ${ }^{3}$ (1) \\ Gabriel Pereira de Oliveira Souza 4 (1) \\ Vitória Suyane Ferreira da $\mathrm{Cruz}^{5}$ (1) \\ Yago Alves Lima 6 (1) \\ Carlos José Oliveira de Matos $^{7}$ (1)
}

\begin{abstract}
${ }^{1}$ Autor para correspondência. Universidade Federal de Sergipe (Lagarto). Sergipe, Brasil. fisiolucianoxavier@hotmail.com 2-7Universidade Federal de Sergipe (Lagarto).Sergipe, Brasil.davidmendonca.s@hotmail.com, debora-sant@outlook.com, gabri_box@hotmail.com, suyane.q@gmail.com, allvesyaago@gmail.com, cjomatos@yahoo.com.br
\end{abstract}

RESUMO | INTRODUÇÃo: O status funcional do indivíduo com Doença Pulmonar Obstrutiva Crônica (DPOC), considerado um indicador preditivo de agravo do quadro clínico e qualidade de vida dessa população. OBJETIVO: Analisar a força muscular, morbidade, qualidade de vida e distância percorrida em pacientes com DPOC e comparar com o grupo não DPOC. MÉTODOS: Foram avaliados 58 voluntários, 29 do grupo DPOC, classificados em GOLD II e 29 o grupo não DPOC, com os seguintes parâmetros mensurados: função pulmonar; variáveis antropométricas; força muscular; morbidade mediante o índice Body mass index, airway Obstruction, Dyspnea, and Exercise capacity (BODE); qualidade de vida com o questionário do Saint George's Respiratory Questionnaire (SGRQ); capacidade pulmonar através da distância percorrida no teste de caminhada de seis minutos. Para análise estatística, realizado o teste de Shapiro Wilk e os testes de Mann-Whitney ou t de Student, assim como o teste de correlação de Pearson, para valores de $p<0,05$. RESULTADOS: A idade média total foi de $61 \pm 7$ anos, a força de extensores de joelho (p:0,0004), com valores de força menores para o grupo DPOC 110,61 $\pm 41,69$; em comparação com do grupo não DPOC 156,31 $\pm 44,09$, destaca-se ainda a força de flexores de cotovelo ( $p$ 0,004), com 96,34 $\pm 26,15$ para grupo DPOC, em comparação ao grupo não DPOC $118,19 \pm 34,71$. Na distância percorrida do TC6M o grupo DPOC percorreu uma média de $274 \pm 107,86$ e o grupo não DPOC $384,98 \pm 100,47(p<0,05)$. O BODE apresentou correlação de $(r=-0,79)$ com a distância percorrida no TC6M e de $(r=0,72)$ com o Saint George total. CONCLUSÕES: Os pacientes com DPOC apresentaram redução da força muscular de membros superiores, extensores de joelho e da distância percorrida, ao comparar com o grupo não DPOC.

PALAVRAS-CHAVE: Doença pulmonar obstrutiva crônica. Força muscular. Teste de Caminhada. Morbidade. Qualidade de vida.
ABSTRACT | INTRODUCTION: The functional status of individuals with Chronic Obstructive Pulmonary Disease (COPD), considered a predictive indicator of clinical condition and quality of life of this population. OBJECTIVE: To analyze muscle strength, morbidity, quality of life and distance traveled in patients with COPD and compare compare them with the group non-COPD. METHODS: Fifty-eight volunteers were evaluated, 29 from the COPD group, classified as GOLD II and 29 in the non-COPD group, with the following parameters measured: pulmonary function; anthropometric variables; muscle strength; morbidity through the Body mass index, airway Obstruction, Dyspnea, and Exercise capacity (BODE); quality of life with the Saint George's Respiratory Questionnaire (SGRQ); lung capacity through the distance covered in the six-minute walk test. For statistical analysis, the Shapiro Wilk test and the Mann-Whitney or Student $t$ tests were performed, as well as the Pearson correlation test, for $p$ values $<0,05$. RESULTS: The mean total age was $61 \pm 7$ years, the strength of knee extensors ( $p$ 0.0004), with lower strength $(\mathrm{N})$ values for the COPD group 110,61 $\pm 41,69$; compared to the non-COPD group $156,31 \pm 44,09$, the strength of elbow flexors ( $p$ 0,004) stands out, with $96,34 \pm 26,15$ for the COPD group, compared to the non-COPD group $118,19 \pm 34,71$. In the distance covered in the 6MWT, the COPD group covered an average of $274 \pm 107,86$ and the non-COPD group $384,98 \pm 100,47(p<0,05)$. The BODE presented a correlation between $(r=-0,79)$ and the distance covered in the 6MWT and $(r=0,72)$ with the total Saint George. CONCLUSIONS: Patients with COPD presented a reduction in muscle strength of the upper limbs, knee extensors and the distance covered, when compared with the non-COPD group.

KEYWORDS: Chronic obstructive pulmonary disease. Muscle strength. Walk test. Morbidity. Quality of life. 


\section{Introdução}

A doença pulmonar obstrutiva crônica (DPOC), caracterizada por sintomas respiratórios persistentes e limitação do fluxo de ar devido as alterações nas vias aéreas e/ou alveolares geralmente causadas por exposição significativa a partículas ou gases nocivos e destruição parenquimatosa, cujas contribuições relativas variam de acordo ao indivíduo?.

No Brasil, ocorrem cerca de 40 mil mortes a cada ano, sendo que a cada hora, três brasileiros morrem em decorrência da $\mathrm{DPOC}^{2}$. O impacto epidemiológico, clínico e socioeconômico da DPOC ainda está em constante aumento, além disso deverá ser a terceira principal causa de morte no mundo até 2030. Considerada uma das principais causas de morbidade e mortalidade em todo o mundo, a DPOC induz a uma carga econômica e social substancial e crescente ${ }^{1}$.

A inflamação crônica causa estreitamento das pequenas vias aéreas e destruição do parênquima pulmonar, desencadeando perda de acessos alveolares nas pequenas vias aéreas na qual diminui o recuo elástico pulmonar. Por sua vez, essas mudanças diminuem a capacidade das vias aéreas de permanecerem abertas durante a expiração. Entre os sintomas, o mais característico da DPOC é a dispneia e muitos fatores são envolvidos em seu mecanismo, incluindo limitação do fluxo aéreo, armazenamento de gases, anormalidades de troca de gás, hipersecreção mucosa, disfunção muscular respiratória e disfunção do músculo esquelético ${ }^{1,2}$. Com a progressão da doença, os pacientes sofrem de deterioração do status funcional e limitações em atividades de vida diária. O estado funcional prejudicado é evidenciado como preditivo de exacerbações, internações hospitalares e mortalidade ${ }^{3}$.

Com a progressão, também ocorre redução da concentração de fibras musculares do tipo I (contração lenta), consequentemente o indivíduo perde força muscular e endurance no músculo fadigando mais rápido ${ }^{4}$. Dessa maneira, a disfunção muscular é representada pela fraqueza, redução da resistência e presença de fadiga muscular, com prevalência de $50 \%$ em pessoas com DPOC grave ou muito grave 5 . O destaque entre estas características é um nível substancialmente reduzido de atividade física ${ }^{4,5}$.
Considerando a causa multifatorial na fraqueza muscular, além da diminuição da funcionalidade que a DPOC gera durante sua progressão, desencadeando alterações na morbidade, qualidade de vida e até na capacidade funcional, este estudo objetivou-se analisar a força muscular, morbidade, qualidade de vida e distância percorrida em pacientes com DPOC, e comparar com o grupo não DPOC.

\section{Métodos}

Trata-se de um estudo transversal, no período de Fevereiro à Maio de 2018, onde foram incluídos 58 pacientes de ambos os sexos, com idade entre 49 e 77 anos. Estes foram divididos em 2 grupos: 0 primeiro composto por indivíduos com diagnóstico clínico de DPOC com obstrução ao fluxo de ar de moderada (GOLD II), estabelecido pelas normas da Global Initiative for Chronic Obstructive Lung Disease (GOLD)'; Grupo não DPOC e não tabagistas, sem lesões pulmonares, após avaliação clínica do pneumologista, compondo assim o grupo controle.

Os critérios de inclusão foram: ser paciente ambulatorial do serviço de pneumologia do Hospital Universitário da Universidade Federal de Sergipe, Aracaju-SE, Brasil; ter diagnóstico clínico de DPOC há pelo menos 6 meses; constar com exame de espirometria no prontuário feito, no mínimo 3 meses antecedentes; apresentar estabilidade clínica, ter 40 anos ou mais; não apresentar infecções e exacerbações nos últimos 3 meses e ausência de alterações osteoneuromusculares que limitassem a marcha. Foram excluídos aquele que tenham sofrido fratura, lesões ou traumas que pudessem interferir na avaliação da força muscular, assim como aqueles que passaram por cirurgias abdominais e torácicas recentemente.

A pesquisa foi aprovada sob o CAAE: 80590617.3.0000.5546, do Comitê de Ética em Pesquisa da Universidade Federal de Sergipe, parecer $n^{\circ} 2.434 .504$. Todos os pacientes foram informados com relação aos procedimentos e assinaram um Termo de Consentimento Livre e Esclarecido (TCLE) antes de sua participação no estudo. 
Entre as variáveis estudadas, o índice de massa corporal (IMC) foi calculado por meio da equação peso (kg) /estatura² (m). A capacidade funcional pulmonar foi avaliada por meio da espirometria, através do espirômetro (Minispir), sendo possível observar o volume expiratório forçado no primeiro segundo (VEF1) e a relação VEF1/ Capacidade Vital Forçada (CVF), mensurado no ambulatório de pneumologia.

Na sequência, foram submetidos à avaliação da força dos músculos: bíceps braquial, tríceps, flexores do punho e quadríceps, realizada no membro dominante informado pelo paciente e quantificada através do dinamômetro portátil MicroFET ${ }^{\circledR} 2$ (Hoggan Health Industries, West Jordan, UT, EUA), modo High Threshold. A unidade da variável de força obtida em Newton (N). O paciente foi posicionado sentado numa cadeira com apoio dos braços, mantendo o ângulo de $90^{\circ}$ de quadril e joelhos, com as mãos apoiadas na cadeira. Com o dinamômetro posicionado na porção distal do membro, o paciente realizava o movimento resistindo à força do avaliador nos últimos graus de movimento e mantendo por 3 segundos. Foram realizados 3 movimentos no membro dominante, com o tempo de descanso de 30 segundos entre cada um deles ${ }^{6}$. O maior valor foi considerado o pico de força (PF).

O teste de força foi realizado pelo mesmo avaliador, com encorajamento verbal padronizado e vigoroso durante a manobra, com o objetivo de estimular o paciente a desempenhar um esforço máximo durante todo o tempo de contração muscular e o avaliador estava atento às compensações e manobra de valsalva.

Posteriormente, realizada avaliação da morbidade através do índice Body Mass-Index, Airflow Obstruction, Dyspnea and Exercise Capacity (BODE). $O$ índice BODE de cada indivíduo foi calculado com base na combinação de quatro variáveis, com as seguintes pontuações: uma medida de composição corporal, o IMC, de 0 a 1 ponto; uma medida da intensidade de obstrução ao fluxo aéreo (VEF1\% previsto pré-broncodilatador), de 0 a 3 pontos; uma medida da sensação subjetiva de dispneia (escala do MRC), de 0 a 3 pontos e uma medida da capacidade de exercício (distância percorrida no TC6M), de 0 a 3 pontos. A pontuação final do índice BODE varia de 0 a 10 pontos, sendo que, quanto maior o valor do índice, pior a condição do paciente ${ }^{8}$.
A avaliação subjetiva da sensação de dispneia foi feita por meio da escala MRC, para composição do índice BODE. Validada em língua portuguesa e composta por apenas cinco itens, dentre os quais o paciente escolhe $o$ item que corresponde a quanto a dispneia limita sua vida diária7,8.

Aplicado o Questionário do Hospital Saint George na Doença Respiratória (SGRQ), para avaliar qualidade de vida relacionada à saúde ${ }^{9,10}$. Este instrumento é específico para doenças respiratórias crônicas, constituído de 76 itens e dividido em três domínios: 1) Sintomas: relacionado às queixas dos problemas respiratórios, (pontuação máxima: 662,5); 2) Atividades: relacionado às atividades que normalmente têm provocado falta de ar nos últimos dias, (pontuação máxima: 1209,1); e 3) Impacto: relacionado ao controle da doença e interferência nas atividades funcionais, (pontuação máxima: 2117,8 ). O total é a soma de todas as questões e seções do questionário e sua pontuação máxima é de 3989,4.

Para a capacidade física, realizado o teste de caminhada de 6 minutos (TC6M) de acordo com as diretrizes estabelecidas pela American Thoracic Society ${ }^{11}$. Executado num terreno de 30 metros, plano e sem obstáculos, pelo mesmo avaliador com experiência. Os dados vitais como pressão arterial sistêmica, frequência cardíaca, frequência respiratória, nível de dispneia (Escala de Borg) e saturação de oxigênio foram aferidos antes e depois do teste. Foi pedido ao paciente que caminhasse de um extremo ao outro da pista, com a maior velocidade possível, durante os seis minutos sob acompanhamento do avaliador.

Caso o paciente sentisse sintomas como dores em membros inferiores, taquicardia ou qualquer outro sintoma de desconforto o teste era interrompido imediatamente. A sequência dos testes realizados foi estabelecida buscando o menor desgaste físico do voluntário. Iniciando com a avaliação da força, descanso de 5 minutos, seguindo com o TC6m. Para calcular a distância predita no TC6m foi utilizada a fórmula: $890,46-(6,11 \times$ idade $)+\left(0,0345 \times\right.$ idade $\left.^{2}\right)+(48,87 x$ gênero $)-(4,81 \times \mathrm{IMC})$ [masculino $=1$, feminino $=0$ ]; proposta por Brito et al. ${ }^{12}$. 


\section{Análise estatística}

Os dados foram apresentados através de média e desvio padrão ( $\pm D P$ ). As distribuições das variáveis numéricas foram analisadas inicialmente pelo teste de normalidade de Shapiro-Wilk, após esta análise, os dados foram comparados entre o grupo DPOC e o grupo controle através do teste t de Student ou Mann-Whitney, de acordo com o teste de normalidade. Para as correlações foi utilizado o coeficiente de Pearson ( $r)^{7}$, classificando em: Nula (0), fraca $(0$ a 0,3$)$, regular $(0,3$ a 0,6$)$, forte $(0,6$ a 0,9$)$ ou muito forte $(0,9$ a 1$)$. $O$ software utilizado para análise estatística foi o Bioestat 5.3 e sendo considerado nível de significância de $95 \%(p<0,05)$.

\section{Resultados}

Na amostra de 58 indivíduos sendo 29 indivíduos não DPOC e 29 com diagnóstico de DPOC classificados como obstrução ao fluxo de ar moderada, GOLD II e descritos demograficamente na Tabela 1. A idade média foi de $61,46 \pm 8,94$ anos e índice de massa corpórea (IMC) com média de $25.87 \pm 4.84 \mathrm{~kg} / \mathrm{m}^{2}$. Previsivelmente o grupo dos saudáveis foi mais ativo quanto ao nível de atividade física e de capacidade funcional avaliado pelo TC6M.

O grupo DPOC obteve no TC6M uma distância inferior $(274,51 \pm 107,86)$ não somente ao seu predito $(405,03$ ( $p$ $=0,05)$ ), mas também ao Grupo Controle $(384,98 \pm 100,47)$. Sendo assim, evidencia um melhor rendimento físico dos indivíduos do Grupo Controle $(p=0,001)$.

Tabela 1. Caracterização demográfica e clínica da amostra DPOC moderado e o grupo não DPOC no período de fevereiro a maio de 2018

\begin{tabular}{lccc}
\hline & DPOC $(\mathbf{n = 2 9 )}$ & Não DPOC $(\mathbf{n = 2 9 )}$ & p \\
\hline Idade & $64,24 \pm 8,33$ & $58,68 \pm 8,79$ & 0,04 \\
IMC $\left(\mathrm{kg} / \mathrm{m}^{2}\right)$ & $24,17 \pm 4,81$ & $27,58 \pm 4,30$ & 0,004 \\
Gênero $(\mathrm{h} / \mathrm{m})$ & $12 / 17$ & $6 / 23$ & - \\
Sedentarismo \% (n) & $89,66(26)$ & $41,38(12)$ & - \\
Tabagismo \% (n) & $37,93(11)$ & $0(0)$ & - \\
Etilismo \% $(\mathrm{n})$ & $6,89(2)$ & $13,79(4)$ & - \\
Exposição gases tóxicos, \% $(\mathrm{n})$ & $68,96(20)$ & $10,34(3)$ & - \\
TC6M & $274,51 \pm 107,86$ & $384,98 \pm 100,47$ & 0,001 \\
VEF1(Pré broncodilatador(L)) & $1,11 \pm 0,25$ & - & -
\end{tabular}

h: homens; m: mulheres; IMC: índice de massa corpórea; TC6M: teste de caminhada de seis minutos; VEF1(L): volume expiratório forçado no primeiro minuto, em Litros. Dados apresentados em frequência absoluta, média \pm dp e utilizado teste $\mathrm{t}$ Student para comparação das variáveis.

Tabela 2. Comparação da Força Muscular DPOC e o grupo não DPOC no período de fevereiro a maio 2018

\begin{tabular}{lcc}
\hline Grupo muscular & DPOC & Grupo Não DPOC \\
\hline Flexores de Cotovelo (N) & $96,34 \pm 26,15$ & $118,19 \pm 34,71$ \\
Extensores de Cotovelo (N) & $74,14 \pm 23,86$ & $94,09 \pm 33,05$ \\
Flexores de Punho (N) & $72,53 \pm 19,79$ & $88,47 \pm 21,41$ \\
& & $0,01 *$ \\
Extensores de Joelho (N) & $110,61 \pm 41,69$ & $156,31 \pm 44,09$
\end{tabular}

*: Valores < 0,05. N: Força muscular em Newton; teste t de Student para comparação das variáveis. 
Houve diferença estatística, tabela 2, entre o grupo DPOC e grupo Não DPOC, em relação a força muscular em todos os grupos musculares, em destaque os extensores de joelho ( $\mathrm{p}, 0004)$. Grupo DPOC foram avaliados para

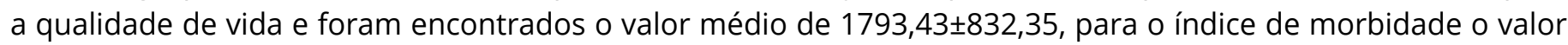
de 4,79 $\pm 2,65$ (Tabela 3).

Tabela 3. Apresentação de média e desvio padrão do Questionário Saint George e Índice BODE, em DPOC e o grupo não DPOC no período de fevereiro a maio de 2018

\begin{tabular}{lcc}
\hline & Grupo DPOC & Valor máximo de referência \\
\hline Sintomas & $298,07 \pm 173,23$ & 662,5 \\
Atividade & $644,77 \pm 331,49$ & 1209,1 \\
Impacto & $849,38 \pm 422,03$ & 2117,8 \\
Saint George total & $1793,43 \pm 832,35$ & 3989,4 \\
Índice BODE & $4,79 \pm 2,65$ & 10,0 \\
\hline
\end{tabular}

Foi encontrada uma correlação entre o índice BODE e apresentou uma correlação positiva forte com o domínio de atividade do Saint George $(r=0,73)$, com Saint George total $(r=0,72)$; correlação negativa forte com volume expiratório forçado no primeiro segundo (VEF1) $(r=-0,82)$; com o TC6M $(r=-0,79)$. O Saint George apresentou uma correlação negativa moderada com VEF1 $(r=-0,57)$ e com o TC6M $(r=-0,56)$ (tabela 4).

Tabela 4. Correlação entre as variáveis primárias em DPOC e o grupo não DPOC no período de fevereiro a maio de 2018

\begin{tabular}{lc}
\hline VARIÁVEIS & $\mathbf{r}^{*}$ \\
\hline VEF1 X BODE & $-0,82$ \\
TC6M X BODE & $-0,79$ \\
Flexores de Cotovelo X BODE & $-0,24$ \\
Extensores de Cotovelo X BODE & $-0,20$ \\
Flexores de Punho X BODE & $-0,51$ \\
Extensores de Joelho X BODE & $-0,27$ \\
Sintomas X BODE & 0,50 \\
Atividade X BODE & 0,73 \\
Impacto X BODE & 0,65 \\
Saint George(total) X BODE & 0,72 \\
Saint George(total) X TC6M & $-0,56$ \\
Saint George(total) X VEF1 & $-0,57$ \\
\hline
\end{tabular}

*r= valor da correlação entre as variáveis; Coeficiente de Pearson para correlação das variáveis.

\section{Discussão}

Foi encontrado nesse estudo perda de força muscular e declínio da capacidade funcional em pacientes DPOC, GOLD II. Estudos demonstram que o sedentarismo apresenta-se como um importante fator de risco modificável, estando presente em $78,3 \%$ dos pacientes ${ }^{13,14}$, ao encontro deste estudo, em que a porcentagem de sedentarismo encontrada nos pacientes com DPOC foi de $89,66 \%$, logo o grupo com DPOC apresentou-se predominantemente sedentário, apenas $10 \%$ dele realiza algum tipo de atividade física regular. Enquanto $58 \%$ dos voluntários não DPOC relataram a prática de atividade física regular, sugere-se que devido aos fatores como a presença de dispneia, o paciente DPOC reduz a prática de atividade física ocasionando consequências como a fraqueza muscular tanto de membros inferiores quanto membros superiores. 
As características sociodemográficas dos pacientes com DPOC II no presente estudo foram semelhantes a outras pesquisas6, corroborando, com a frequência de DPOC-GOLD || entre indivíduos com mais de 65 anos de idade e com história de tabagismo significativa, a justificativa plausível, no presente estudo foi por ter sido amostra de conveniência.

Autores monstram que o processo inflamatório sistêmico, juntamente com o estresse oxidativo na DPOC, desencadeiam diversas alterações de bioenergética e estruturais da musculatura esquelética, seu desfecho, corrobora com nossos resultados que mostra a redução da força dos músculos dos membros superiores quando comparado com o grupo não DPOC, e consequentemente gera uma baixa capacidade de exercício e menor desempenho muscular nos pacientes com DPOC 6 .

Outro estudo ${ }^{15}$, avaliou a força do quadríceps em 75 pacientes no estágio IV da DPOC (classificação GOLD), em que $92 \%$ dos participantes tiveram diminuição da força muscular do quadríceps e $76,5 \%$ destes classificavam-se com obstrução do fluxo aéreo leve a moderada, diferentemente deste estudo, por ser realizado apenas em DPOC no estágio II. Todavia, os resultados demostraram que ambos os grupos apresentaram-se com boa força muscular considerando o valor médio predito de 101.56 para o grupo DPOC e de 93.86 para o grupo não DPOC.

Estes resultados indicam um declínio funcional, implicando em um histórico de perda de peso, que geralmente representa perda de massa muscular ${ }^{15}$, é observado nessa população que a perda de força muscular observada nas fases iniciais da doença precede o início dos sintomas que induzem a redução do condicionamento nas atividades diárias ${ }^{15,16}$. A fraqueza muscular periférica é decorrente à atrofia por desuso, provocada pelo descondicionamento físico predominantemente encontrada nos $\mathrm{MMIII}^{15,17}$, na qual favorece impactos funcionais principalmente nas atividades de vida diária (AVD's), e maior limitação em desenvolvê-las. Além disso, outros sintomas como redução da capacidade pulmonar, dispneia, hiperinsuflação dinâmica e fatores psíquicos relacionados ao medo da fadiga tornam o indivíduo predisposto à fraqueza muscular ${ }^{18}$.
Quanto a força muscular de membros superiores, sabe-se que a força muscular distal de punho pode ser preservada, levando em consideração que os pacientes com DPOC são capazes de continuar a realizar atividades de vida diária que envolvem movimentos repetidos do punho, incluindo agarrar, segurar e carregar objetos ${ }^{19}$, portanto, importante para o desenvolvimento das atividades de vida diária, visto ser uma musculatura que está sendo recrutada na maioria das atividades.

O IMC apresenta relação de dependência com força muscular, em que o índice de massa corpórea baixo apresenta maior probabilidade do indivíduo evoluir para fraqueza muscular ${ }^{18,20}$. A afirmação pode explicar o resultado da pesquisa em apresentar média do pico de força de quadríceps superior ao valor predito. Em relação a força muscular do membro superior, o grupo controle apresentou média do pico de força maior do que o grupo DPOC, pois a média de IMC da amostra DPOC foi de $24.17 \mathrm{~kg} / \mathrm{m}^{2}$, enquanto que a média de IMC do grupo controle foi de $27.58 \mathrm{~kg} / \mathrm{m}^{2}$.

Quanto ao TC6M, os resultados da pesquisa confirmaram que os pacientes com DPOC percorrem uma menor distância comparados aos saudáveis. Dentre os possíveis motivos, sugere-se o sedentarismo, por apresentar característica forte nesse grupo, corroborando com o estudo de Hernandes et al. ${ }^{21}$, ao observar que indivíduos que possuem a doença passam maior parte do tempo sentados ou deitados, como forma de evitar o sintoma da dispneia, além de caminharem com menor intensidade de movimento. Os autores sugerem que o fato possa ser atribuído ao estilo de vida adotado por ele como consequência da doença. O contrário também é afirmado por outros autore $^{20,21}$, em que a inatividade pode ser precursora das alterações sistêmicas e não consequência da gravidade da doença.

Entretanto pesquisas ${ }^{21}$ propõem que o TC6M é mais adequado para capacidade funcional quando comparado ao $\mathrm{VEF}_{1}$. Em nosso estudo o grupo com DPOC foi mais inativo e consequentemente percorreu menor distância no TC6M. Ao comparar a distância percorrida no TC6M obtido com o predito foi observada uma baixa distância percorrida em toda a amostra traduzindo por redução significativa da capacidade física, 
com um déficit de $29 \%$, ao encontro de outros estu$\operatorname{dos}^{21,22}$ que indicam que a redução da tolerância ao exercício físico é um indicador significativo de mortalidade e pior qualidade de vida na doença pulmonar obstrutiva.

Quanto a idade, pesquisas ${ }^{21,22}$, confirmam que quanto maior a idade, menor será a distância percorrida. Quanto ao $\mathrm{IMC}^{27}$ observou-se que idosos com índice $<25 \mathrm{Kg} \cdot \mathrm{m}^{2}$ caminharam maior distância em metros que os voluntários com índice $>25 \mathrm{~kg} / \mathrm{m}^{2}$. Contrapondo-se aos resultados desta pesquisa, em que a amostra obteve uma média de IMC $=25 \mathrm{Kg} / \mathrm{m}^{2}$, mesmo assim os dois grupos tiveram baixo resultado no TC6M quando comparados com o predito.

Quanto ao índice BODE e suas correlações funcionais, a variável que melhor correlacionou foi o VEF1 $(r=-0,82)$. O BODE mostrou forte correlação com o escore total do SGRQ $(r=0,72)$ e também com o domínio de atividade do escore do SGRQ $(r=0,73)$, mas apresentou correlação moderada $(r=0,50)$ com o domínio sintoma, corroborando com outros estudos23,24. A qualidade de vida é prejudicada em pacientes com DPOC e se deteriora com o aumento da gravidade da doença que está associado a um aumento significativo no escore do SGRQ.

Além disso, estudos ${ }^{23,24,25}$ trazem que um índice de tabagismo maior, afeta a qualidade de vida dos indivíduos com DPOC, especialmente com os sintomas dos pacientes e o escore de impacto, que descreve o estado psicológico dos pacientes. Outro estudo mostrou que o domínio Atividades que mede a dificuldade em fazer as atividades físicas diárias, demonstrando como a limitação funcional respiratória se associa negativamente com a realização destas tarefas cotidianas e consequentemente na qualidade de vida destes pacientes resultou no maior escore percentual médio de $80,4 \pm 7,2^{25}$.

Como fatores limitantes do nosso estudo, a amostra foi selecionada por conveniência, não sendo representativa da população total; o número de pacientes distribuídos por sexo não foi similar entre os grupos. Destaca-se um (n) reduzido em comparação com outros estudos, devido a recusa de alguns pacientes a fazer parte da pesquisa.

\section{Conclusão}

Os pacientes com DPOC avaliados apresentaram redução da força muscular de flexores e extensores de cotovelo, flexores de punho, extensores de joelho e menor distância percorrida quando comparados com o grupo não DPOC.

\section{Contribuições dos autores}

Silva DM participou da concepção e planejamento, coleta de dados, revisão crítica do conteúdo e aprovação da versão final do manuscrito. Santos DN, Souza GPO e Cruz VSF participaram da coleta de dados, elaboração da primeira versão, revisão crítica do conteúdo e aprovação da versão final. Gomes LX participou da concepção e planejamento, coleta, análise e interpretação dos dados, elaboração da primeira versão, revisão crítica do conteúdo e aprovação da versão final do manuscrito. Matos CJO participou da concepção e planejamento, análise e interpretação dos dados, elaboração da primeira versão, revisão crítica do conteúdo e aprovação da versão final.

\section{Conflitos de interesses}

Nenhum conflito financeiro, legal ou político envolvendo terceiros (governo, empresas e fundações privadas etc.) foi declarado para nenhum aspecto do trabalho submetido (incluindo, mas não se limitando a subvenções e financiamentos, participação em conselho consultivo, desenho de estudo, preparação de manuscrito, análise estatística, etc.).

\section{Referências}

1. Gold 20120. Glob Inititiative Chronic Obstr Lung Dis. [Internet]. 2020. Available from: https://goldcopd.org/

2. Brasil. Ministério da Saúde. Portaria SAS/MS no 609, de 06 de junho de 2013, retificada em 14 de junho de 2013. Protocolo Clínico e Diretrizes Terapêuticas: Doença pulmonar obstrutiva crônica. [cited 2020 Jan 30]; Available from: https:// portalarquivos2.saude.gov.br/images/pdf/2014/abril/02/pcdtdoenca-pulmonar-obs-cronica-livro-2013.pdf

3. Liu Y, Li H, Ding N, Wang N, Wen D. Functional Status Assessment of Patients With COPD. Medicine (Baltimore). 2016;95(20):e3672. doi: 10.1097/MD.0000000000003672

4. Silva ALG, Garmatz E, Carvalho LL, Cardoso DM, Paiva DN. Handgrip and functional capacity in Chronic Obstructive Pulmonary Disease patients. Fisioter Mov. 2017;30(3):501-7. doi: 10.1590/1980-5918.030.003.a008 
5. Santos K, Karloh M, Gulart AA, Munari AB, Mayer AF. Relação entre força muscular periférica e respiratória e qualidade de vida em pacientes com doença pulmonar obstrutiva crônica. Med (Ribeirão Preto). 2015;48(5):417-24. doi: 10.11606/issn.2176-7262. v48i5p417-424

6. Nyberg A, Saey D, Maltais F. Why and how limb muscle mass and function should be measured in patients with chronic obstructive pulmonary disease. Ann Am Thorac Soc. 2015;12(9):1269-77. doi: 10.1513/AnnalsATS.201505-278PS

7. Pearson K, Fisher RA, Henry FI. Karl Pearson and R. A. Fisher on Statistical Tests: A 1935 Exchange from Nature. The American Statistician. 1994;48(1):2-11. doi: 10.2307/2685077

8. Celli BR, Cote CG, Marin JM, Casanova C, Oca MM, Mendez RA et al. The Body-Mass Index, Airflow Obstruction, Dyspnea, and Exercise Capacity Index in Chronic Obstructive Pulmonary Disease. N EngI J Med. 2004;350(10):1005-12. doi: $10.1056 /$ NEJMoa021322

9. Jones PW, Quirk FH, Baveystock CM. The St George's Respiratory Questionnaire. Respir Med. 1991;(Suppl):25-31. doi: 10.1016/ s0954-6111(06)80166-6

10. Sousa TC, Jardim JR, Jones P. Validação do Questionário do Hospital Saint George na Doença Respiratória (SGRQ) em pacientes portadores de doença pulmonar obstrutiva crônica no Brasil. J Pneumol. 2000;26(3):119-28. doi: 10.1590/S010235862000000300004

11. Crapo RO, Casaburi R, Coates AL, Enright PL, Maclntyre NR, McKay RT et al. ATS statement: Guidelines for the six-minute walk test. Am J Respir Crit Care Med. 2002;166(1):111-7. doi: 10.1164/ ajrccm.166.1.at1102

12. Britto RR, Probst VS, Andrande AF, Samora GA, Hernandes NA, Marinho PE et al. Reference equations for six-minute walk distance based on a Brasilian multicenter study. Braz J Phys Ther. 2013;17(6):556-63. doi: 10.1590/S1413-35552012005000122

13. Giacomelli IL, Steidle LJM, Moreira FF, Meyer IV, Souza RG, Pincelli MP. Pacientes portadores de DPOC hospitalizados: análise do tratamento prévio. J Bras Pneumol Brasília. 2014;40(3):229-37. doi: 10.1590/S1806-37132014000300005

14. Landal AC, Monteiro F, Hevely BCS, Kanesawa LM, Hernandes N, Pitta F. Fatores associados à melhora da composição corporal em indivíduos com DPOC após treinamento físico. Fisioter em Mov. 2014;27(4):633-41. doi: 10.1590/0103-5150.027.004.AO15

15. Kharbanda S, Ramakrishna A, Krishnan S. Prevalence of quadriceps muscle weakness in patients with COPD and its association with disease severity. Int J Chron Obs Pulmon Dis. 2015;10:1727-35. doi: 10.2147/COPD.S87791

16. Bone AE, Hepgul N, Kon S, Maddocks M. Sarcopenia and frailty in chronic respiratory disease: Lessons from gerontology. Chron Respir Dis. 2017;14(1):85-99. doi: 10.1177/1479972316679664
17. Shrikrishna D, Patel M, Tanner RJ, Seymour JM, Connolly BA, Puthucheary ZA et al. Quadriceps wasting and physical inactivity in patients with COPD. Eur Respir J. 2012;40(5):1115-22. doi: $\underline{10.1183 / 09031936.00170111}$

18. Man WD-C, Soliman MG, Nikoletou D, Harris ML, Rafferty GF, Mustfa N et al. Non-volitional assessment of skeletal muscle strength in patients with chronic obstructive pulmonary disease. Thorax. 2003;58(8):665-9. doi: 10.1136/thorax.58.8.665

19. Polkey MI, Rabe KF. Chicken or egg: Physical activity in COPD revisited. Eur Respir J. 2009;33(2):227-9. doi: $\underline{10.1183 / 09031936.00176808}$

20. Aquino ES, Peres TM, Lopes IBV, Cantro FMR, Coelho CC, Cunha Filho IT. Correlação entre a composição corporal e força, resistência Da Musculatura respiratória e capacidade de Exercício em pacientes com doença pulmonar obstrutiva crônica. Fisioter e Pesqui. 2010;17(1):58-62. doi: 10.1590/S1809-29502010000100011

21. Hernandes NA, Teixeira DC, Probst VS, Brunetto AF, Ramos $\mathrm{EM}$, Pitta F. Profile of the level of physical activity in the daily lives of patients with COPD in Brazil. J Bras Pneumol. 2009;35(10):94956. doi: $10.1590 / s 1806-37132009001000002$

22. Garcia-Rio F, Rojo B, Casitas R, Lores V, Madero R, Romero $D$ et al. Prognostic value of the objective measurement of daily physical activity in patients with COPD. Chest. 2012;142(2):338-46. doi: 10.1378/chest.11-2014

23. Hill K, Gardiner P, Cavalheri V, Jenkins S, Healy G. Physical activity and sedentary behaviour: applying lessons to chronic obstructive pulmonary disease. Intern Med J. 2015;45(5):474-82. doi: $\underline{10.1111 / i m j .12570}$

24. Sarka SK, Das SK, Choudhury S, Datta S, Sarkar S, Basuthakur $S$ et al. Evaluation of correlation of BODE index with healthrelated quality of life among patients with stable COPD attending a tertiary care hospital. Lung India. 2015;32(1):24-8. doi: $\underline{10.4103 / 0970-2113.148434}$

25. Pôncio TGHO, Carvalho DS, Randow RM. Avaliação da qualidade de vida de pacientes em oxigenoterapia domiciliar em um município da zona da mata mineira. Il Jornada de Iniciação Científica da FACIG. 2017; Minas Gerais: Centro Universitário UNIFACIG;2017. P. 1-5. 